\title{
Much Polyphony but Little Harmony: Otto Sackur's Groping for a Quantum Theory of Gases
}

\author{
Massimiliano Badino $^{1}$ and Bretislav Friedrich ${ }^{2}$
}

The endeavor of Otto Sackur (1880-1914) was driven, on the one hand, by his interest in Nernst's theorem, statistical mechanics, and the problem of chemical equilibrium, and, on the other, his goal to shed light on classical mechanics from the quantum vantage point. Inspired by the interplay between classical physics and quantum theory, Sackur chanced to expound his personal take on the role of the quantum in the changing landscape of physics in the turbulent 1910s. In this paper, we tell the story of this enthusiastic practitioner of the old quantum theory and early contributor to quantum statistical mechanics, whose scientific ontogenesis provides a telling clue about the phylogenesis of his contemporaries.

Key words: Otto Sackur, Richard Abegg, Fritz Haber, Max Planck, Ludwig Boltzmann, Max Born, Peter Debye, Walther Nernst, Otto Stern, Josiah Willard Gibbs, Arnold Eucken, Jacobus Henricus van't Hoff, Cato Maximillian Guldberg, Peter Waage, Hugo Tetrode, affinity, heat theorem/third law of thermodynamics, quantization, phase space, entropy, quantum theory of gases, quantum statistical mechanics, black body radiation, heat capacity, Sackur-Tetrode equation, law of mass action, chemical constant, Kaiser Wilhelm Institute for Physical Chemistry and Electrochemistry, University of Breslau, First Solvay Conference.

\footnotetext{
${ }^{1}$ Max-Planck-Institut für Wissenschaftsgeschichte, Boltzmannstraße 24, D-14195 Berlin, Germany. Current address: Centre d'Història de la Ciència, Facultat de Ciències, Universitat Autonoma de Barcelona, Cerdanyola del Valles, 08193 Bellaterra (Barcelona), Spain

${ }^{2}$ Fritz-Haber-Institut der Max-Planck-Gesellschaft, Faradayweg 4-6, D-14195 Berlin, Germany
} 


\section{Introduction}

Upon its inception within the context of radiation theory, the quantum hypothesis rapidly diffused into other fields. By 1910, the unravelling of the classical traditions of physics and chemistry in the light of the quantum became increasingly evident. Quantum theory was catapulted to the fore by the First Solvay Conference (1911), in the wake of which many leading physicists embraced the quantum hypothesis as a key to solving outstanding problems in the theory of matter.

Until about 1910, quantum physics had drawn much of its inspiration from two sources. The first was the complex formal machinery connected with Max Planck's theory of radiation and, above all, its close relationship with probabilistic arguments and statistical mechanics. The fledgling 1900-1901 version of this theory hinged on the application of Ludwig Boltzmann's 1877 combinatorial procedure, which Planck used to determine the state of maximum probability for a set of oscillators. In his 1906 book on heat radiation, Planck made the connection with gas theory even tighter. In order to illustrate the use of the procedure that Boltzmann had originally developed for an ideal gas, Planck showed how to extend the analysis of the phase space, commonplace among the practitioners of statistical mechanics, to electromagnetic oscillators [1]. In doing so, Planck identified a crucial difference between the phase space of the gas molecules and that of the oscillators that had been featured in quantum theory. Whereas in Boltzmann's statistical mechanics a state corresponds to an arbitrarily small cell of the phase space, defined by a system's coordinates and momenta, the quantum hypothesis requires a partition of phase space into elementary regions - or volume elements - each equal to Planck's constant, $h$. As we will see below, phase space quantization enabled extending the quantum hypothesis to systems whose complexity exceeded that of the oscillators. However, by 1906 Planck did not draw any conclusion about the phase space of gas molecules.

The other source of early quantum physics was Albert Einstein's 1907 theory of solids, the first successful application of the quantum hypothesis outside of the realm of heat radiation [2]. By assuming that the particles of the solid behave like oscillators whose energy is given by Planck's radiation formula, Einstein was able to derive a temperature dependence of the solid's specific heat that agreed qualitatively with Nernst's theorem (see below). Ein-

stein's theory demonstrated that the quantum hypothesis could be used to solve outstanding 
problems in the theory of matter. Among its descendants are the nearly definitive theories of the thermal properties of solids developed by Peter Debye (1912) and by Max Born \& Theodore von Karman (1912-13).

From the close link between the gas and the oscillators mentioned above, one may get the impression that the leap to the quantum theory of a gas was a fairly intuitive process. However, this was not the case, not the least because of conceptual difficulties: its advanced formal apparatus notwithstanding, the quantum hypothesis was conceived for periodic systems characterized by a frequency. A swarm of whirling particles, a gas was thus viewed as the opposite of a paradigmatic quantum system. For instance, Nernst, in 1911, resolutely denied the possibility of quantizing translation [3], although he had advocated the extension of quantum theory to gases. ${ }^{3}$ Furthermore, there was no experimental motivation for extending the quantum hypothesis to a gas. As late as 1913, Arnold Eucken pointed out that no genuine quantum phenomenon had yet been observed involving such a system [4].

No wonder then that at that time the quantum theory of a gas was more of a concern to outsiders, such as Sackur, rather than to experts, such as Einstein or Planck. The first attempts to develop a quantum theory of a gas thus came about in response to concerns about peripheral issues, namely chemical equilibria, instead of arising from an interest to revamp the kinetic theory or thermodynamics.

Indeed, the emergent quantum framework compelled a host of young researchers to approach the problem of a gas with a pragmatic attitude: without an interest or resources to partake in the big foundational debates of the time, they ventured to squeeze out from the nascent quantum theory an essence that could serve the specific goals of their research. This pragmatic attitude spurred a variety of applications of the quantum that, to a modern eye, may appear gross, sloppy, and naive, but that sometimes led to genuine progress.

Herein, we discuss one remarkable example of such a pragmatic approach, namely Otto Sackur's exploits in the quantum theory of gases. Sackur's work was discussed in previous historical accounts; ${ }^{4}$ in this paper, however, we delve deeper into his theories, with the aim to uncover aspects of his style of work which was representative of a part of the quantum community. A characteristic trait of this style is Sackur's use of quantum theory as a tool to tackle a problem deeply rooted in classical physics, namely the chemical equilibrium in

\footnotetext{
${ }^{3}$ On this point see [5].

${ }^{4}$ Mentions of Sackur's work can be found in $[6,7]$.
} 
gaseous systems. Sackur's goal-oriented attitude was partly responsible for some of the formal and conceptual inaccuracies and contributed to making his papers look tentative. However, his bold attempt to deploy the quantum hypothesis across classical statistical mechanics would prove instrumental for preparing Planck's path to his own theory of quantum gases.

Sackur's work developed along two non-orthogonal directions: it was driven, on the one hand, by his interest in the Nernst theorem, statistical mechanics, and the problem of chemical equilibrium, and, on the other, his goal to shed light on classical mechanics from a quantum vantage point. Inspired by the interplay between classical physics and quantum theory, Sackur chanced to expound his personal take on the role of the quantum in the changing landscape of physics in the 1910s. In this paper, we tell the story of this enthusiastic practitioner of the old quantum theory and early contributor to quantum statistical mechanics.

\section{Biographical Overview}

Otto Sackur was born in Breslau (now Wrocław), Silesia, on September 29, 1880. ${ }^{5}$ He studied chemistry, first at the University of Breslau, whose Chemistry Department, headed by Albert Ladenburg (1842-1911) since its establishment in 1897, belonged to the most prestigious in Germany. In Breslau, Sackur found an enlightened mentor, Richard Abegg (1869-1910), who introduced him to modern physical chemistry. Sackur further advanced his chemistry education at Heidelberg and Berlin before receiving his doctorate from Breslau on July 31, 1901.

Sackur's academic career at Breslau took a detour, first via the Kaiserliches Gesundheitsamt in Berlin (from October 1902 till October 1904), where he worked under the direction of Theodore Paul on problems related to public health. Subsequently, he joined William Ramsey's laboratory at the University College London (from October 1904 until March 1905) and then Walther Nernst's brand new laboratory at the Berlin University (from March 1905 until September 1905). During the latter stays he became privy to the most up-to-date work in physical chemistry.

Upon his return to Breslau in October 1905, he obtained his Habilitation and the title

\footnotetext{
${ }^{5}$ There are only few sources available about Sackur's life. Here we have especially relied on [8] and obituaries written by colleagues and friends $[9,10,11]$.
} 


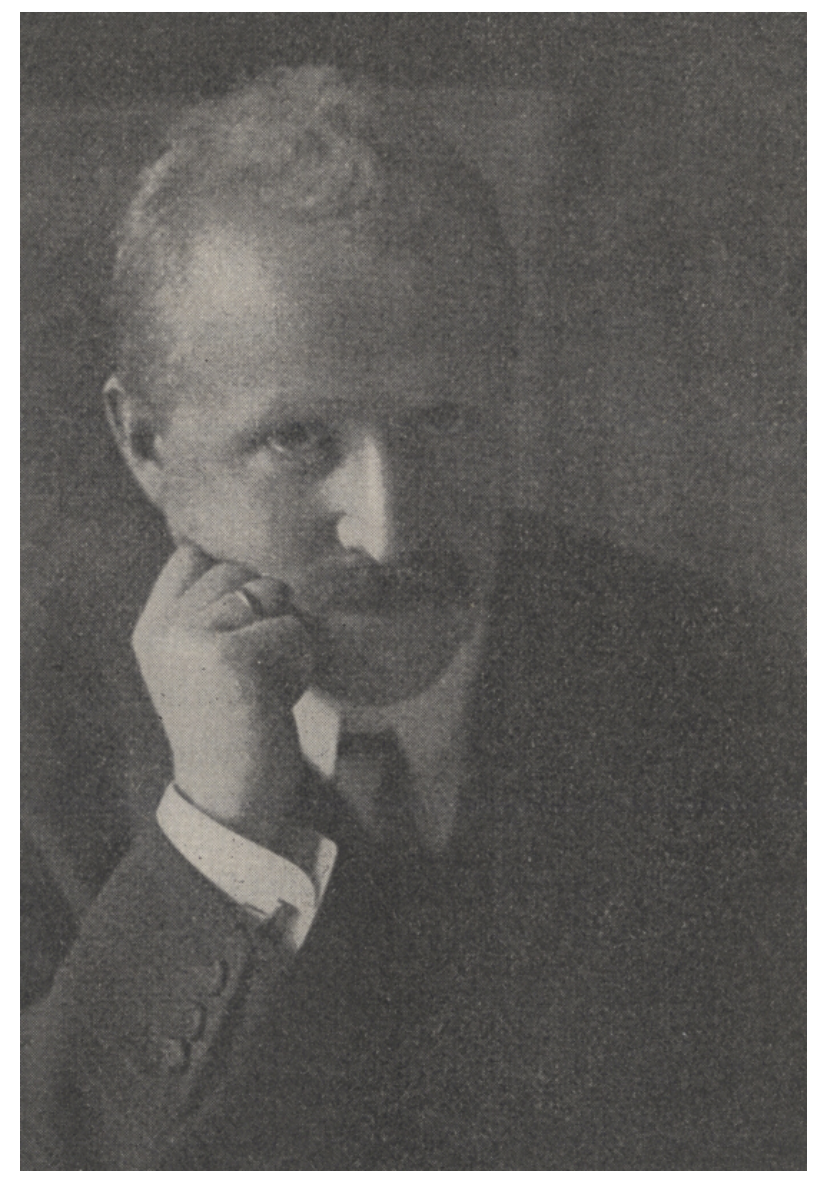

Figure 1: Otto Sackur, 1880-1914

of Privatdozent. For some years, he taught at Breslau and worked side by side with Abegg, with an eye on a more secure position. Sackur's hopes were shattered by two unfortunate events. In 1909 Albert Ladenburg had retired because of poor health (he died two years later) and the new director of the department, the 1907 Nobel Prize winner Eduard Buchner (1860-1917), a fermentation biochemist, had little sympathy for physical chemistry. A year later, Richard Abegg died tragically in a ballooning accident. ${ }^{6}$ Without an academic sponsor and a laboratory, Sackur had to rely on his pedagogical skills to survive. He accepted minor teaching assignments, devised a course of chemistry for dentists, and wrote textbooks on thermodynamics, ${ }^{7}$ while trying desperately to keep abreast of the latest de-

\footnotetext{
${ }^{6}$ In Tessin, near Rostock [12, 13].

${ }^{7}$ During this period, Otto Stern earned his PhD under Sackur's supervision, on osmotic pressure of "generalized soda-water," a topic of his own choice [14]. Also, it was Sackur who, using in turn Fritz
} 
velopments in physical chemistry. It was during this period of existential difficulties that Sackur had launched his research at the intersection of physical chemistry, thermodynamics, and quantum theory, in the hope of a reward - a more senior academic appointment.

His hopes were fulfilled at the end of 1913, when, thanks in part to a mediation by Clara Immerwahr, Fritz Haber's first wife and Abegg's former student, Sackur received a call to Haber's Kaiser-Wilhelm-Institut für Physikalische Chemie und Elektrochemie in Berlin [17]. In 1914 he was promoted to the rank of a department head. After the outbreak of the Great War, Sackur was enlisted in military research at Haber's institute, but, on the side, succeeded in carrying on with his experiments on the behavior of gases at low temperatures. In connection with their efforts to develop new and more effective explosives and propellants, Sackur, jointly with Haber and Gerhard Just, attempted to replace the liquid tear gas in artillery shells with a substance that would act as both irritant and propellant. The choice was cacodyl chloride, which Bunsen had first synthesized in 1837, but which chemists had scarcely researched since, because it was such a powerful irritant and toxin. On 17 December 1914, during an experiment intended to improve further the effectiveness of the cacodyl chloride, Sackur was killed by an explosion at his work bench. He was just 34 years old [18].

\section{The Problem of Chemical Equilibrium}

In the course of the nineteenth century, the concept of a chemical equilibrium underwent several transformations. Around 1850, the old notion that a reaction is at equilibrium when all the "chemical forces" involved are balanced out was gradually replaced by a kinetic view: a (reversible) chemical reaction never stops completely, but only reaches a stationary state when the reaction rates in the forward and backward direction become equal.

The ratio between the forward and backward rates was termed the equilibrium constant, K. In 1864 Cato Maximilian Guldberg (1836-1902) and Peter Waage (1833-1900) discovered that the equilibrium constant depended on the ratio of the concentrations of the reactants and products ('law of mass action'). Their pioneering paper, published originally in Norwegian, was largely ignored until 1877 when Wilhelm Ostwald (1853-1932) adopted the law and corroborated its validity by his own experiments. ${ }^{8}$ Jacobus Henricus van't Hoff (1852-1911)

Haber's mediation, helped Stern to his post-doctoral position with Albert Einstein, in 1912 [15]. On Sackur's pedagogical activity see [16].

${ }^{8} \mathrm{~A}$ collection of the publications of Guldberg and Waage on the topic was later published in the series 
discovered the law independently in 1884 and two years later derived a formula that governs the temperature dependence of $K[20]$.

A second breakthrough in the study of chemical equilibria occurred in the 1880s when thermodynamics was applied to chemical problems. As early as 1878, Josiah Willard Gibbs (1839-1903) used the concept of the maximum work produced by a reaction to define chemical equilibrium: a reaction has reached equilibrium when it produced all the work it was capable of producing [21]. Curiously, Gibbs's insight faced a fate similar to that of Guldberg and Waage's, as its publication in an obscure journal likewise hampered its dissemination. In 1882, Hermann Helmholtz (1821-1894) arrived at an equivalent result independently. He distinguished between a bound and a free energy of a system. The latter is transformed into work during a chemical reaction, whose equilibrium is reached when the free energy drops to its minimum value [22]. As Eucken paraphrased it:

[T]he (maximum) work represents a quantity whose knowledge leads immediately to the solution of the [equilibrium problem]: only when a chemical process is able to perform work, it will go on spontaneously. Hence, one can also say in what direction [a reaction] will go, if it is given for which transformation [...] the maximum work has a positive sign. If in a chemical transformation no work is produced, then the system is in a state of equilibrium [23].

The concept of free energy amounted to a well-founded overhaul of the old intuitive notion of 'affinity.' The internal energy of a chemical system is made up of free energy, which can be used to produce work, and of bound energy (also called the 'latent heat of reaction') which cannot. At equilibrium, a relation exists between free energy and the equilibrium constant. This relation can be used to evaluate the equilibrium constant provided the free energy is known. Helmholtz found a way to obtain the free energy by combining the first and second laws of thermodynamics for the case of an isothermal and isochoric gaseous reaction (i.e., one taking place at a constant temperature and volume). Such a combination leads to an equation whose integration at constant volume yields the free energy in terms of the internal energy and an integration constant [24]. The problem of calculating the equilibrium constant was thus reduced to determining the reaction energy (i.e., the difference between the internal energy of the reactants and products) and the integration constant. Experimentally, this task Ostwald Klassiker der Exakten Wissenschaften in a translation by Richard Abegg [19]. 
was all but easy and much effort was expended in the final decades of the nineteenth century at collecting the data needed to evaluate the free energy for various substances. ${ }^{9}$

The Heat Theorem, enunciated by Walther Nernst (1864-1941) in 1906, amounted to a third breakthrough. ${ }^{10}$ Nernst observed that as the absolute temperature $T$ approaches zero, the slopes of the temperature dependence of the free energy and of the internal energy tend asymptotically to zero, without concluding that internal energy and free energy themselves become equal at $T=0$. Nevertheless, based on his observation, Nernst was able to set the free energy integration constant to zero and thereby made it possible to find, at any temperature $T$, the free energy from the internal energy. The internal energy could, in turn, be determined from thermochemical data $[31,32]{ }^{11}$

However, the experimental data as well as the theoretical arguments that supported Nernst's assumptions held true only for condensed-matter systems and could not be extrapolated to a gas. ${ }^{12}$ The treatment of the equilibrium of gaseous reactions appeared to be beyond the reach of Nernst's theorem. In response, Nernst devised an ingenious detour which made it possible to evaluate the equilibrium constant of gaseous reactions after all. He noticed that the integration constant of the van't Hoff equation, which concerns the temperature dependence of the equilibrium of gases or dilute solutions, was closely related to the integration constant of the Clausius-Clapeyron equation, which governs the temperature dependence of the pressure of a vapor in phase equilibrium with a liquid or solid condensate (the two equations are isomorphic). Nernst dubbed the integration constant of the van't Hoff equation the chemical constant [31, p. 22]. The chemical constant had to be determined experimentally for each gaseous species involved in the reaction. Once the chemical constants of reactants and products were available, the van't Hoff equation yielded the equilibrium constant of a gaseous reaction in terms of the reaction energy and the heat capacity at constant pressure.

Hence what was still needed were the chemical constants. In order to evaluate the chemi-

\footnotetext{
${ }^{9}$ For theoretical and experimental aspects of physical chemistry at the end of the nineteenth century, see $[25,26],[27]$ and [28].

${ }^{10}$ Julius Thomsen (1826-1909) in 1852 and Marcellin Berthelot (1827-1907) in 1869 had speculated on the behavior of chemical reactions in the vicinity of absolute zero. See [27, 29]. For Nernst's personal take on the story see the opening chapter of [30].

${ }^{11}$ For a modern treatment see [33].

${ }^{12}$ For a thorough presentation of the issue see [34, 30].
} 
cal constants, Nernst resorted to various approximations of the Clausius-Clapeyron equation with the goal of establishing the dependence of the chemical constants on thermochemical parameters and then determining the constants from there [34, pp. 55-76]. However, this strategy was only partially successful and, by 1911, the chemical constants became available for only a handful of substances. This was chiefly due to the unreliability of the approximations involved and to the difficulties encountered when performing measurements at cryogenic temperatures.

Sackur followed a different strategy. In 1911 he commented:

Only Nernst's theorem makes it possible to establish the [chemical] constant [...] which determines the chemical behavior of gases from measurements on

pure substances (vapor pressure of liquids or solids) and thereby [...] also the entropy constant [35].

The reference to the entropy constant is key: Sackur recognized that the vapor-condensate system can be dealt with by applying the second law of thermodynamics, which leads to a simple relation between the integration constant of entropy and the chemical constant. This relation is at the core of Sackur's 1911 advance: he set out to calculate the entropy constant by invoking statistical mechanics. This was a bold move not only because of the rather tentative status of the statistical approach at the time but also because entropy itself was perceived as difficult to define and, moreover, wedded to 'mysterious' applications of probability. Physical chemists of the time preferred the concept of maximum work to characterize chemical equilibrium. In the most influential physical chemistry textbook of the time, Nernst's Theoretische Chemie, entropy is mentioned only to be brushed away as dispensable. However, Sackur's move had not remained unrewarded: he was able to derive the first quantum-statistical expression for the entropy of an ideal gas (in the limit of high temperatures and low densities). A similar expression was discovered independently at about the same time by Hugo Tetrode (1895-1931) and is known as the Sackur-Tetrode equation. The Sackur-Tetrode equation made it possible to evaluate the entropy constant and thus the chemical constant in terms of better known quantities.

\section{The Beginnings of the Quantum Theory of Gases}

During the first decade of the 20th century, many proponents of the quantum hypothesis, 
such as Nernst, Max Planck (1858-1947), and Albert Einstein (1879-1955), had come to think that kinetic theory, quantum theory, the heat theorem, statistical mechanics, and (physical) chemistry were all closely related. Einstein's 1907 quantum theory of solids exemplifies the power of the quantum in treating what used to be called the kinetic theory of matter while Nernst, at about the same time, became convinced that the quantum hypothesis implied the validity of his theorem for gases. ${ }^{13}$ On a more general level, it became apparent that the quantum called for new ways of applying statistical mechanics to both the theory of radiation and the theory of matter.

Presumably, Sackur got to know about these developments at the scientific meetings, which he had attended tirelessly in those years. ${ }^{14}$ The approach to the theory of matter based on sophisticated mathematical techniques - which quantum theory entailed - was exactly his cup of tea. Unlike the majority of physical chemists of the time who had an aversion to the formal complications of kinetic theory (including major protagonists such as Nernst), Sackur was convinced that physical chemistry could not do without the most advanced mathematical tools available. During his academic career in Breslau, he taught classes in both kinetic theory (summer semesters 1910 and 1912) and in the "mathematical treatment of chemistry' (winter semester 1906 and summer semester 1908). Sackur's lecture notes became the basis for an acclaimed book written jointly with Abegg, which was even translated into English [41].

In Sackur's usage, the term 'kinetic theory' did not refer to the nineteenth century analysis of collisions leading to the riddle of irreversibility. Instead, 'kinetic theory' meant an attempt to trace back the thermal behavior of systems, chemical or other, to the arrangements of their molecules. This is illustrated by his first paper dedicated to such problems. ${ }^{15}$

\footnotetext{
${ }^{13}$ Although Nernst was reluctant to commit his conviction to paper, there are at least two pieces of indirect evidence: First, in about 1910 Nernst attempted to prove his theorem on the grounds of a purely thermodynamic argument independent of the nature of the substances involved [36]; Second, after 1906 he put his Berlin group to work on the measurement of the specific heats of gases. Before the Solvay Conference, Nernst supervised three doctoral dissertations on the specific heats of gases - by F. Voller (1908), F. Keutel (1910), and R. Thibaut (1910). At the same time, Mathias Pier worked on the measurement of specific heats at high temperatures [37, 38], while Fritz Koref and Eucken concentrated on low temperatures [39, 40].

${ }^{14}$ From Physikalische Zeitschrift and Zeitschrift für Elektrochemie it can be established that Sackur attended the meetings of the Bunsen Society for Physical Chemistry from 1906 until 1914, the year of his death. In 1908, he also reported about the meeting for Physikalische Zeitschrift.

${ }^{15}[42]$. Attempts along a similar direction were [3, 43, 44].
} 
Sackur argued that with decreasing temperature, the available molecular energy becomes increasingly restricted, limiting the number of 'cells' (states) over which the molecules can be distributed. When the temperature drops close to zero, the number of 'cells' tends to one, as a result of which the distribution becomes ordered: all the molecules end up herded in the last remaining 'cell.' Since entropy $S$ depends on the number of molecular arrangements, $W$, via the Boltzmann principle, $S=k \ln W$, it was apparent that $S=0$ for $W=1$ when all molecules are in the same cell. Since Nernst's theorem amounts to saying that entropy approaches zero as the temperature goes to zero [45], Sackur concluded that the third law reduces to the claim that low-temperature molecules are arranged in their energy space in the most orderly fashion.

The main sources of inspiration for Sackur's paper [42] were precisely works mentioned in the introduction: Planck's 1906 Wärmestrahlung as well as Einstein's 1907 theory of solids [2]. Sackur was especially intrigued by the way in which Einstein intertwined the classical statistico-mechanical approach with the new quantum hypothesis. In the second part of his paper, Einstein put forward a second proof of the heat theorem, by showing that the quantum hypothesis, in the case of a solid, implied that the entropy constant is zero. For Sackur, the transition from the gas to the solid was justified by Einstein's general procedure:

[A] complete kinetic theory of the solid state of aggregation might perhaps be built on Einstein's presuppositions just as van der Waals' theory was built on the presuppositions of the classical gas theory [42].

Furthermore, Sackur's paper attests to its author's internalization of Planck's concept of absolute entropy. Classical thermodynamics defines only an entropy difference and leaves the integration constant undetermined. But Planck was convinced that entropy expresses a fundamental property of nature and, therefore, must have an absolute value. Quantum theory combined with the heat theorem affirmed that entropy's integration constant can be set to zero. ${ }^{16}$

Sackur adopted the concept of absolute entropy and used it as a building block of his new quantum theory of the ideal gas. In his second 1911 paper, he tried to construct a general application of his peculiar version of kinetic theory to chemical problems. Here 'application'

\footnotetext{
${ }^{16}$ Planck dwelled on the concept of absolute entropy both in the third edition of his Thermodynamik and in the second edition of the Wärmestrahlung [45, 46].
} 
is the key word that expresses the second direction of Sackur's work: his attempt to transform abstract procedures into potent treatments of classical problems. To Sackur, the glue that holds together quantum theory, kinetic theory, and physical chemistry is the concept of probability, whose value had been well proven in the course of the investigations of gases but that could be applied to other problems as well:

This idea has been hitherto applied only to the variations of state of an ideal gas, that is to physically and chemically uniform substances, as well as to radiation phenomena. Now it seems that it can be applied to any spontaneously occurring processes in nature, for example to chemical reactions and to irreversible processes of any kind that are connected with an increase of the probability of a closed system. This generalization of the concept of probability to chemically distinct states of matter appears to me a simple consequence of Boltzmann's view $[35]$.

Henceforth, Sackur set out to find a general way of comparing the probability of different states. The case of chemical equilibrium in an ideal gas was particularly suitable for this aim, as it went just a step beyond Boltzmann's gas theory.

In his attack on the problem of entropy from the 'kinetic' side, Sackur follows explicitly Planck's statistico-mechanical treatment of an ideal gas based on the partitioning of the phase space. In classical statistical mechanics, the state of the gas as a whole depends on the number of molecules in each possible mechanical state, the latter being determined by the positions and velocities of the molecules. If we imagine a space whose coordinates are the molecular positions and velocities, we can represent each mechanical state as an 'elementary region' in this space. Remarkably, since in classical mechanics all infinitesimal states are possible, the 'volumes' of these elementary regions are in turn infinitesimal and equal. If we have $n_{1}$ molecules in the first state, $n_{2}$ molecules in the second and, in general, $n_{i}$ molecules in the $i$-th state, the gas as a whole can be described by the 'state distribution' $n_{1}, n_{2}, \ldots, n_{i}, \ldots$ of such 'occupation numbers' of each region.

In 1877, Boltzmann proposed a simple recipe for calculating the probability $W$ of such a distribution, namely by counting the number of ways in which one can permute the molecules between different regions without changing the overall distribution. Sackur made use of Boltzmann's formula and plugged it into the Boltzmann principle relating $W$ to entropy. 
The resulting expression for entropy then depended on the occupation numbers. In order to eliminate this dependence, Sackur evaluated the maximal probability of the state distribution under the constraint of constant energy and the number of molecules, ${ }^{17}$ which corresponds to equilibrium. Thereby, Sackur arrived at an expression for the translational entropy of a monoatomic gas which contained an explicit dependence on the volume of the 'elementary regions.' A comparison with the thermodynamic expression for the entropy of an ideal gas yielded a handy formula for the entropy constant in terms of the universal gas constant, the molecular weight, and the volume of the 'elementary regions'.

So far, Sackur's argument only used well-established elements of kinetic theory and statistical mechanics. Among the ingredients contributing to the entropy constant, only the volume of the elementary region could not be specified within the framework of classical physics. Hence a determination of the entropy constant, and therefore of the chemical constant, called for a closer investigation of the concept of an 'elementary region.' So what were Sackur's 'elementary regions'?

\section{Sackur's 'Elementary Regions'}

A considerable part of Sackur's paper [35] deals with the properties of the 'elementary regions.' First of all, he points out that the volume of the elementary region must have a well-defined value because the entropy constant is related to the equilibrium constant, which is indeed a constant for any given reaction. Sackur regarded this requirement as a hint that the issue crosses beyond the boundaries of classical physics:

[S]ince pure kinetic [theory] has hitherto not been able to say anything about the magnitude of [the elementary volume], there is a gap in the sequence of inferences that lead from the kinetic hypothesis to the equation of state of a gas [35].

Sackur, however, did not immediately suggest that the fixed volume of an elementary region is determined by Planck's quantum of action, $h$. There might be a purely technical

\footnotetext{
17 in doing so, Sackur invoked the Stirling formula and replaced summation by integration. On the one hand, the applicability of the Stirling formula relies on the assumption that the elementary regions are large enough to contain many molecules. On the other hand, replacing summations by integration requires the regions to be infinitesimal. Classical kinetic theory hinges upon the balance between these two contravening requirements, see also $[47,48]$. We will discuss Sackur's reflections on it in the next section.
} 
reason for his reluctance to do so. Following Boltzmann and Planck, Sackur had worked with the position-velocity space whose volume element did not have the dimension of action. In fact, Sackur carried out the dimensional analysis of the entropy constant, but he limited himself to verifying that it depended on the volume of the gas in the expected fashion. Although noncommittal as to its physical meaning, the peculiarity of a fixed-magnitude elementary volume did not escape Sackur's attention. He sought to give both a visual and a conceptual interpretation of this result.

Visually, the fixed-magnitude volume seems to entail the fact that the molecules do not pass smoothly from one state to another or, equivalently, that they do not spread out into the entire space allowed: ${ }^{18}$

The gas molecules do not distribute themselves uniformly over the whole allowed space and they cannot have all possible velocities from zero to infinity, but [...] they concentrate around individual points in space, like bullets on a target, and $[\ldots]$ their velocity components change in jumps [35].

Here we encounter a characteristic trait of Sackur's approach. Drawing on the visualizability of a discrete space, Sackur initially presumes a discontinuous behavior of the molecules. But in reality his underlying picture always maintained the aspect of a provisional sketch. As we will see in the next section, he eventually dropped discontinuity and moved toward an operational approach which sidestepped precise commitments about the microscopic nature of the gas particles. This was not only due to the objective difficulties of framing a clear picture of the quantum phenomena, but went hand in hand with the problem-oriented character of his program. The motivation behind his research was the idea of putting the quantum in the service of classical problems - and not developing an understanding of the quantum's nature.

Conceptually, Sackur relied on Planck and traced the 'atomicity' of the 'elementary region' back to the calculation of probability. In his 1900 paper where he enunciated the quantum hypothesis, Planck had argued that an actual computation of a state's probability

\footnotetext{
${ }^{18}$ To a modern reader, Sackur's talk about a tendency of molecules to clump in phase regions may suggest some, albeit naive, anticipation of quantum statistics [6]. However the main problem that quantum statistics had to deal with, namely the extensivity of entropy, was not considered here. And when the problem came up, Sackur did not seem to recognize it as a fundamental issue.
} 
is possible only if the total energy is divided into elements of a finite size. The 'atomicity' of energy then followed directly from the Boltzmann relation between entropy and probability. Sackur had subscribed to Planck's view unconditionally:

[E]ither [we] postulate the physical reality of a finite elementary region (and of finite elementary quanta) or [we] give up the unequivocal relation between entropy and probability [35].

Sackur's prime interest, however, lay in the physico-chemical properties of the elementary regions. Coming to terms with what the elementary volume means is the theme of the second part of his analysis, whose conclusion is twofold: First, the elementary volume is not a universal constant, but must, somehow, depend on the molecular mass. Sackur argued that if the elementary volume were the same for all gases with the same number of molecules, then the chemical constant would have to decrease with increasing molecular weight. This, however, would contradict Nernst's experiments, which had in fact found the inverse behavior.

Second, Sackur concluded that the elementary volume depends on the number of molecules. By making use of an ingenious argument,${ }^{19}$ Sackur contended that the probability of an ideal gas consisting of $N$ molecules partitioned into $q$ subsystems with $N^{\prime}$ molecules each and probability $W_{N^{\prime}}$ has a total probability $W_{N}=W_{N^{\prime}}^{q}$. An application of Boltzmann's principle then led Sackur to the conclusion that the ratio between the elementary volume and the number of molecules $N$ - and not the elementary volume alone - is a universal constant. Sackur's result contains a grain of truth in that it renders the entropy in his formula extensive. ${ }^{20} \mathrm{In}$ his Wärmestrahlung Planck started from extensivity and the law of the composition of probabilities to derive the Boltzmann principle [1]. Apparently Sackur did not fully realize the significance of his own argument and result, but Planck did. Three years later, while struggling with the problem of making quantum entropy extensive, Planck would take up Sackur's condition by stating that the elementary volume of the phase space of a gas depended on the number of molecules in the gas [49].

\footnotetext{
${ }^{19}$ From this argument one may discern another source of inspiration in Sackur's work. For it closely resembles the 1905 light-quantum paper in which Einstein compares the probabilities for a gas to be in a given volume and in a part of that same volume.

${ }^{20}$ If $S_{1}=k \ln W_{1}$ and $S_{2}=k \ln W_{2}$ are the entropies of two subsystems and $W_{12}=W_{1} W_{2}$ is the probability of the composed system, then $S_{12}=S_{1}+S_{2}$, which is the definition of extensivity of entropy.
} 
Sackur's paper is representative of the general concerns of his work at large. As stated in the title, Sackur's prime interest was the 'application' to physical chemistry of the methods developed by Planck in radiation theory. Sackur followed Planck closely in making use of probability and in handling the state space, but stopped short of introducing explicitly the quantum hypothesis. Instead, he attempted to clarify the properties of the elementary volume as a means to calculate theoretically the chemical constants. In the concluding section of the paper, Sackur tested his procedure on the dissociation of iodine molecules and was able to retrieve the correct formula for the mass-action law.

\section{The Generalization of Sackur's Statistical Theory}

Sackur's focus on the burning issues of physical chemistry deflected his attention from the impact that his method could have on quantum theory at large. Tetrode, whose work approached the problems of fledgling quantum statistics from a more abstract viewpoint, stressed the quantum interpretation of the elementary volume as well as the problem of extensivity right at the outset [50]. ${ }^{21}$ However, Sackur himself realized, on a careful reconsideration, that his procedure opened up an intriguing prospect. He explored this prospect in a paper included in the Nernst Festschrift, published in May $1912 .^{22}$ In that paper, the notion of the quantum of action and its application to the calculation of the chemical constants appeared in the very title.

Sackur first tackled the problem of extensivity. He realized that the dependence of the elementary volume on the number of molecules was necessary to guarantee extensivity of the entropy function, but was no longer happy with the way it came about in his previous paper. At this point, Sackur was already convinced that the essence of the quantum hypothesis was probabilistic. As a consequence, he thought that a reconciliation of quantum theory with thermodynamics relied on a suitable definition of probability. In order to appreciate his procedure, we will briefly summarize Sackur's peculiar concept of probability.

In 1911, Sackur defined the probability of a state as

... the number that measures how many times a $[\ldots]$ state $[\ldots]$ is more probable

\footnotetext{
${ }^{21}$ For a discussion of Tetrode's paper, see [6, 7].

${ }^{22}$ The paper [51] is dated March, 1912. Since also Tetrode's article was published in March, it seems that Sackur worked out the consequences of his approach independently.
} 
than a state of the same energy and volume in which all atoms would have the same positions, directions and velocities [35].

This idiosyncratic definition, a result of Sackur's personal reading of Planck and Boltzmann, led to the usual number of possible permutations of a state distribution. To retrieve extensivity, Sackur stated that the definition must be changed and

we must resort [...] to the concept of molecular disorder [51].

Once again, Sackur obtained this concept from the tradition of Planck and Boltzmann, but reinterpreted it in a peculiar way. In an arbitrary state, it is possible that more than one molecule occupies the same energy cell (or phase-space cell). If the state is perfectly disordered, each cell contains exactly one molecule. In other words, the molecules are totally spread out throughout the available energy (or phase) space. This concept of molecular disorder could not be more at odds with Planck's and Boltzmann's, ${ }^{23}$ but it accomplished its intended goal. As a result, Sackur modified the definition of probability as the ratio of the number of favorable cases to the number of possible cases, a proper fraction, and was again able to retrieve the term containing the ratio between the elementary volume and the number of particles in the entropy formula which guarantees its extensivity. This is yet another instance of Sackur's willingness to interpret quite freely the traditions of statistical mechanics in order to arrive at a concrete result.

The second theoretical innovation introduced in the paper is the interpretation of the elementary volume in terms of Planck's constant. Sackur kept the position-velocity space, but relied on Sommerfeld's authority for interpreting the elementary volume as the ratio $h^{3} / m^{3}$, with $m$ the molecular mass. ${ }^{24}$ This expression for the elementary volume enabled

... to calculate this quantity [i.e., the elementary volume] characteristic for the determination of the vapor pressure and of the chemical behavior without any experimental determination [51].

\footnotetext{
${ }^{23}$ Boltzmann considered the molecular disorder as the condition for applying probability in gas theory, while Planck was convinced that disorder had the power to eliminate any violation of the second law of thermodynamics. For a discussion of these two views see [52].

${ }^{24}$ Sommerfeld had proposed the interpretation of the elementary volume in terms of $h$ as a general quantization condition for a periodic system at the First Solvay Conference and in a widely discussed paper [53].
} 
The last part of the paper deals with the calculation of the chemical constants for monoand poly-atomic gases. Sackur displays his familiarity with the experimental data and his ability to work with them. Despite an incorrect expression for the rotational energy, Sackur obtained a reassuring agreement between his theoretical values and the experimental chemical constants, which signaled to him that his formula was correct.

In his subsequent paper [55], Sackur's shift towards an operational approach went the whole way; this paper was the most ambitious of his four articles on gaseous equilibria and statistical mechanics. Apparently, he had not perceived his prior probabilistic argument as satisfactory and thus sought to present a more general analysis. The striking feature of Sackur's original approach based on the partitioning of the state space was that it resolved, with facility, the problem of the quantization of an aperiodic system. As we have noted in the introduction, the quantum hypothesis was introduced to deal with periodic systems and expressed a relationship between the system's energy and characteristic frequency. But there was no obvious way of ascribing a frequency to a gas. In order to circumvent this issue, Sackur worked on the state space of the system rather than the system itself - quantizing the elementary regions instead of the system's behavior. However, in his next paper [55], he changed tack. In accordance with his view that the quantum was intimately connected with probability, he endeavored to bring together the quantum hypothesis and the classical probabilistic procedures used by kinetic theorists of the time. The result was a captivating argument with a nineteenth century flavor.

The argument goes as follows. Sackur first considers the case of a periodic system - a set of particles oscillating around an equilibrium point - which resembles an Einstein solid. There is no assumption about the nature of these particles except that, after a very long time, they take up all possible values of energy. Sackur then tackles the problem of calculating the probability that a particle has an energy between $\epsilon$ and $\epsilon+\Delta \epsilon$ when it passes through the equilibrium point. ${ }^{25}$ Then he envisions a microscopic observer who can watch individual particles and send a signal every time a particle passes through the equilibrium point with the correct energy. As a result, the sought probability is given by the ratio of the number of signals, $n$, to the total number of particles, $N$, i.e., $w=n / N$. This probability, Sackur states, is a function of energy and it is proportional to the width of the cell as well as to the

\footnotetext{
${ }^{25}$ The argument could be carried out for any point of oscillation, but Sackur chooses the equilibrium point since there the energy of the particle is purely kinetic.
} 
span of time over which the observer watches the system, $w=f(\epsilon) \Delta \epsilon \Delta t$. Given that any value of energy is possible, Sackur insisted that it is a fundamental principle of probability calculus that if one waits long enough, all possible events will occur. This principle implies that if $\Delta \epsilon$ decreases, i.e., the energy cells become smaller and more difficult to observe, the same probability can be maintained by extending the observation time $\Delta t$. In other words, $\Delta \epsilon \Delta t=$ constant, a consequence that curiously resembles Heisenberg's energy-time uncertainty relation. ${ }^{26}$

But if the observer is allowed to examine the system for an arbitrarily long time, he will count all the molecules infinitely many times, because they can assume all energy values over a long time. In order to ensure that each molecule is counted only once, the observation time must be set equal to the oscillation period, $\Delta t=\tau$, in which case only molecules with energies $0, \Delta \epsilon, 2 \Delta \epsilon, \ldots$ will be counted when $\Delta \epsilon=\frac{h}{\tau}=h \nu$. The quantum hypothesis thereby affects the energy width $\Delta \epsilon$, that is to say the 'experimental error' that the microscopic observer is allowed to make. The gist of Sackur's probabilistic argument is that the quantum hypothesis is not necessarily a new physical assumption, but rather an 'extension of the old statistical methods:'

Contrary to the old picture developed by Planck for the derivation [of the equations of this theory], it [is] not necessary to assume an atomistic structure of energy or action. It suffice[s] to sharpen the (physical) concept of probability, namely by the almost obvious assumption that the verification of a result is the more probable, the longer one waits for it, and therefore even an extremely unlikely but possible result will have a finite probability to arise after an infinitely long time [55].

Sackur further strived to show that the above quantum constraint emerges as soon as the probabilistic formalism is transformed into a physically workable procedure. The fictitious microscopic observer illustrates precisely this point. His attempt to demonstrate a continuity between the methods of classical statistical mechanics and quantum theory serves two related goals. First, it allows to put aside some of the conundrums that came along with the quantum hypothesis. As the quotation above illustrates, Sackur was more eager to underscore the formal similarities between the classical and quantum approach than the weird consequences

\footnotetext{
${ }^{26}$ The energy-time uncertainty relation has a more genuine predecessor in the work of Niels Bohr [54].
} 
stemming from discontinuity and discretization. Second, the above similarities justified in Sackur's eyes the application of the quantum procedure to the problems left open by the classical theory. Precisely because the quantum hypothesis appeared to him as an extension of statistical mechanics, he expected it to fill the remaining gaps in the thermodynamic and statistico-mechanical treatments of the chemical phenomena.

Sackur then tests his probabilistic argument by retrieving Planck's black-body radiation law and Einstein's formula for the heat capacity of solids at low temperatures. In this connection, Sackur makes an interesting comment, namely that in the limit of high temperatures, one obtains, respectively, the Rayleigh-Jeans formula and the Dulong-Petit law for the two above cases. Then he points out for the case of the Rayleigh-Jeans formula that it is to be ragarded not as a purely classical result but rather an approximate quantum result.

So much for periodic systems. For aperiodic systems, the maximization of entropy proceeds formally in the same way as for periodic ones, except that one has to identify a proper substitute for the characteristic frequency. Sackur argues that the limitation imposed by the observation time must be such that the velocity of the molecule be constant during that time:

$[\mathrm{W}]$ e have to choose the observation time [.. $]$ so that during $\Delta t=\tau$ the molecule experiences no change of velocity, that is no collision [55].

Sackur is apparently referring to the mean time that the molecule spends to cover the mean free path. In that span of time the molecules maintain their velocity and the observer will count only those molecules which are in a given energy cell. ${ }^{27}$

In order to ensure extensivity, Sackur resorted to defining the three components of velocity as the ratio between the corresponding mean free paths and the mean free times. Since the mean free path depends on the number of molecules, this procedure surreptitiously introduces the same dependence as the definition of the elementary cell in the position-velocity space. In effect, Sackur treated the gas as if it consisted of molecules distributed into $V / N$ independent sub-volumes defined by the mean free path.

In the last section of his paper, Sackur extended this approach to polyatomic gases. The classical treatment of the rotational energy he presented contained an error, which Tetrode

\footnotetext{
${ }^{27}$ Sackur's idea of using the mean free path as a parameter to quantize the gas was adopted the following year by Sommerfeld and his collaborator Wilhelm Lenz [56].
} 
pointed out to him in a personal letter. Sackur reacted by publishing a paper dedicated to the discussion of gaseous molecules with two or three atoms, in which he corrected this error. $^{28}$

By the end of 1912, Sackur considered his quantum theory of gaseous equilibrium to be complete: he had calculated the chemical constants for a variety of gases which was his initial stated goal. His contact with Fritz Haber and Haber's Kaiser-Wilhelm-Institut enticed him to work on applications of quantum theory to other physico-chemical problems, such as the behavior of gases at very low temperatures [58]. ${ }^{29}$ This topic preoccupied him during the last, Berlin phase of his career.

\section{Conclusions}

The Sackur-Tetrode equation is perhaps the most lasting of Sackur's contributions to quantum theory and, indeed, to science. It is still presented in textbooks as the quantum expression for the translational entropy of a monoatomic gas at high temperatures and low densities. Like Nernst's heat theorem, it had played an important part in the collective groping that would lead eventually to a complete theory of quantum gases. Sackur's scientific ontogenesis provides a telling clue about the phylogenesis of his contemporaries. Although Sackur's theoretical rendition of the chemical constants was immediately and widely accepted as a touchstone of thermochemistry, his general theoretical reflections did not have the same fate. Vacillations between phase space and microscopic observers, between discontinuity and probabilistic arguments, in a word, between tradition and innovation still reveal deep uncertainties concerning the basis on which his computational results were founded.

Some of his ideas were subsequently adopted or discussed - for instance the $N$ dependence of the elementary cell or the division of the gas volume into $V / N$ subvolumes in order to ensure extensivity. But nobody seems to have followed his suggestion for a purely probabilistic-operational foundation of the quantum hypothesis. Physicists and physical chemists favored Otto Stern's approach based on the vapor-solid equilibrium [60, 61], which

\footnotetext{
${ }^{28}$ [57]; although Tetrode's letter to Sackur has been lost, we know about its existence from a footnote in Sackur's paper.

${ }^{29}$ Because of his intention to move on to the experimental determination of the gas law at very low temperature, Sackur applied for a position in Kamerling Onnes' institute in Leiden. However, Onnes dismissed his application with scorn [59].
} 
eventually triggered Fermi's analysis of the statistics of identical particles [62, 63].

However, reducing Sackur's case to a story about which aspects of his work succeeded to have an impact and which failed to do so would mean to miss more interesting historiographical points. We conclude with two reflections on Sackur and the complex landscape of the quantum physics in the 1910s that he helped to shape.

First, Sackur was a member of the diverse and variegated scientific community that had developed quantum physics. In the 1910 s, the quantum hypothesis made its way into statistical mechanics, radiation theory, spectroscopy, theory of matter, physical chemistry, and atomic modeling. In such a complex landscape, the sheer multitude of the specialists involved lent the contentions about the quantum a considerable polyphony but little harmony. Although formal tools and mathematical techniques were widely shared among the more famous (such as Einstein, Planck, or Nernst) and the relatively unknown (such as Tetrode or Sackur himself) players, the quantum hypothesis meant different things to different people. For instance, Einstein's perspective, shaped mostly by his interest in radiation theory, differed greatly from Nernst's, which depended on his desire to establish the validity of the heat theorem. In Sackur's case, his background in physical chemistry was key to shaping his interpretation of the quantum. Eager to put the quantum to the service of long-standing problems of thermochemistry - as well as to impress a potential employer - Sackur stressed the continuity between classical statistical techniques and the quantum. The variety of approaches and outlooks made the 1910s an interesting period both from a historiographical and an epistemological point of view.

Second, Sackur belonged to a part of the physics community whose research style was quite different from that of, say, Planck, Einstein, or Bohr. Sackur's research was not driven by an adherence to strong principles, as he lacked a grand methodological guidance such as, for instance, Planck's concept of absolute entropy. This difference echoes Suman Seth's recent distinction between 'physics of principle' and 'physics of problems' [64]. Sackur was, no doubt, more sympathetic to an approach of starting from and returning to concrete problems. The state of flux in which quantum physics found itself at the beginning of the 1910s facilitated and almost encouraged problem-oriented lines of attack. Thus, Sackur's was, so to speak, a 'mundane' quantum physics coming 'from below' as a result of his personal understanding of abstract concepts and of his pragmatic agenda. Although curious about the wider implications of his arguments, he remained largely unconcerned about the 
quantum formalism and, in the end, settled on a conservative position, according to which the quantum is an extension of classical statistical mechanics. For a vast majority of Sackur's peers - who would eventually provide a lasting contribution - the quantum was mainly a tool. For them, like for Sackur, quantum physics made sense when viewed in the context of well-defined problems in classical physics that arose within the framework of their own disciplines.

\section{References}

[1] Max Planck, Vorlesungen über die Theorie der Wärmestrahlung (First edition. Lepzig: Barth, 1906), pp. 140-148.

[2] Albert Einstein, "Die Plancksche Theorie der Strahlung und die Theorie der spezifischen Wärme," Annalen der Physik 22 (1907) 180-190.

[3] Walther Nernst, "Zur Theorie der spezifischen Wärme und über die Anwendung der Lehre von den Energiequanten auf physikalisch-chemische Fragen überhaupt," Zeitschrift für Elektrochemie und angewandte physikalische Chemie 17 (1911) 265-275.

[4] Arnold Eucken, "Die Entwicklung der Quantentheorie vom Herbst 1911 bis Sommer 1913," in Arnold Eucken, ed., Die Theorie der Strahlung und der Quanten (Halle: Knapp, 1914), pp. 371-405.

[5] Clayton A. Gearhart, " 'Astonishing successes' and 'bitter disappointment': The specific heat of hydrogen in quantum theory," Archive for History of Exact Sciences 64 (2010) 113-202.

[6] Olivier Darrigol, "Statistics and combinatorics in early quantum theory, II: Early symptoma of indistinguishability and holism," Historical Studies in the Physical Sciences 21 (1991) 237-298.

[7] Agostino Desalvo, "From the chemical constant to quantum statistics: a thermodynamic route to quantum mechanics," Physis 29 (1992) 465-537.

[8] Alexander Kipnis, Entry on Otto Sackur, in Neue Deutsche Biographie, Vol. 22, p. 344 (Berlin: Dunker und Humblot, 2005). 
[9] Friedrich Auerbach "Otto Sackur - Nekrologe," Jahresbericht der Schlesischen Gesellschaft für Vaterländische Kultur 1 (1915) 35-37.

[10] Walther Hertz, "Otto Sackur," Physikalische Zeitschrift 16 (1915) 113-115.

[11] Hans Pick, "Otto Sackur," Chemiker-Zeitung 39 (1915) 13.

[12] Svante Arrhenius, "Richard Abegg," Zeitschrift für Elektrochemie und angewandte physikalische Chemie 16 (1910) 554-557.

[13] Theodor Des Coudres, "Richard Abegg," Physikalische Zeitschrift 11 (1910) 425-429.

[14] Bretislav Friedrich and Dudley Herschbach, "Stern and Gerlach: How a bad cigar helped reorient atomic physics," Physics Today 56 (2003) 53-59.

[15] Thomas S. Kuhn, "Interview with Dr. Otto Stern by Thomas S. Kuhn at Stern's Berkeley home May 29 \& 30, 1962," The Oral History Interviews \& Transcripts Project (American Institute of Physics, 1962).

[16] Massimiliano Badino, "Dissolving the boundaries between research and pedagogy: Otto Sackur's Lehrbuch der Thermochemie und Thermodynamik," in Massimiliano Badino and Jaume Navarro, Research and Pedagogy. A History of Quantum Physics through its Textbooks (Forthcoming).

[17] Jeremiah James, Thomas Steinhauser, Dieter Hoffmann, Bretislav Friedrich, One Hundred Years at the Intersection of Chemistry and Physics: The Fritz Haber Institute of the Max Planck Society 1911-2011 (Berlin: Walter de Gruyter, 2011).

[18] Bretislav Friedrich, Dieter Hoffmann, and Jeremiah James, "One hundred years of the Fritz Haber Institute," Angewandte Chemie International Edition 50 (2011) 10022-10049; Angewandte Chemie 123 (2011) 10198-10225.

[19] Cato M. Guldberg and Peter Waage, "Untersuchungen über die chemischen Affinitäten," Abhandlungen aus den Jahren 1864, 1867, 1879 (Leipzig: Englemann, 1899; translated by Richard Abegg).

[20] Jacobus Van't Hoff, "L'équilibre chimique dans les systeme gezeux, ou dissous á létat dilué," Archives néerlandaises des sciences exactes et naturelles 20 (1886) 239-302. 
[21] Josiah Willard Gibbs, "On the equilibrium of heterogeneous substances," Transactions of the Connecticut Academy 3 (1878) 108-248; 343-524; also in Henry A. Bumstead, ed., The Scientific Papers of Josiah Willard Gibbs, Vol. I, (New York: Longmans, 1906), pp. 55-353.

[22] Hermann Helmholtz, "Die Thermodynamik chemischer Vorgänge," Sitzungsberichte der Berliner Akademie der Wissenschaften 1 (1882) 22-39.

[23] Arnold Eucken, "Der Nernstsche Wärmesatz," Ergebnisse der exakten Naturwissenschaften 1 (1922) 120-162.

[24] Fritz Haber, Thermodynamik technischer Gasreaktionen (Munich: Oldenbourg Verlag, 1905).

[25] Edwin N. Hiebert, "The energetic controversy and the new thermodynamics," in Duane H. D. Roller, ed., Perspectives in the History of Science and Technology (Normal: University of Oklahoma Press, 1971), pp. 67-86.

[26] Edwin N. Hiebert, "Walther Nernst and the application of physics to chemistry," in Rutherford Aris, Howard T. Davis and Roger H. Stuewer, eds, Springs of Scientific Creativity (Minneapolis: University of Minnesota Press, 1983), pp. 203-231.

[27] Diana Kormos Barkan, Walther Nernst and the Transition to Modern Physical Science (Cambridge: Cambridge University Press, 1999).

[28] Patrick Coffey, "Chemical free energies and the third law of thermodynamics," Historical Studies in the Physical Science 36 (2006) 365-396.

[29] Hans-George Bartel and Rudolf Huebener, Walther Nernst, Pioneer of Physics and of Chemistry (Singapore: World Scientific, 2007).

[30] Walther Nernst, Die theoretischen und experimentellen Grundlagen des neuen Wärmesatzes (Halle: Knapp, 1917).

[31] Walther Nernst, "Über die Berechnung chemischer Gleichgewichte aus thermischen Messungen," Nachrichten von der Gesellschaft der Wissenschaften zu Göttingen 1 (1906) 1-39. 
[32] Walther Nernst, "Über die Beziehungen zwischen Wärmeentwicklung und maximaler Arbeit bei kondensierten Systemen," Sitzungsberichte Preussische Akademie der Wissenschaft zu Berlin (1906) 933-940.

[33] Philip M. Morse, Thermal Physics (Second edition. Reading: The Benjamin/Cummings Publishing Company, 1969).

[34] Walther Nernst, Experimental and Theoretical Applications of Thermodynamics to Chemistry (New Haven: Yale University Press, 1907).

[35] Otto Sackur, "Die Anwendung der kinetischen Theorie der Gase auf chemische Probleme," Annalen der Physik 36 (1911) 958-980.

[36] Anne J. Kox, "Confusion and clarification: Albert Einstein and Walther Nernst's Heat Theorem, 1911-1916," Studies in History and Philosophy of Modern Physics 37 (2006) 101-114.

[37] Mathias Pier, "Spezifische Wärme von Gasen bei sehr hohen Temperaturen," Zeitschrift für Elektrochemie 15 (1909) 536-540.

[38] Mathias Pier, "Spezifische Wärme und Gasgleichgewicht nach Explosionversuchen," Zeitschrift für Elektrochemie 16 (1910) 897-903.

[39] Fritz Koref, "Messungen der spezifischen Wärme bei tiefen Temperaturen mit dem Kupferkalorimeter," Annalen der Physik 36 (1911) 49-73.

[40] Arnold Eucken, "Die Molekularwärme des Wasserstoffs bei tiefen Temperaturen," Sitzungsberichte der preussischen Akademie der Wissenschaft (1912) 141-151.

[41] Richard Abegg and Otto Sackur, Physikalisch-Chemische Rechenaufgabe (Leipzig: Barth, 1909).

[42] Otto Sackur, "Zur kinetischen Begründung des Nernstschen Wärmetheorems," Annalen der Physik 34 (1911) 455-468.

[43] Ferenz Jüttner, "Über die Ableitung der Nernstschen Formeln für Reaktionen in kondensierten Systemen," Zeitschrift für Elektrochemie und angewandte physikalische Chemie 17 (1911) 139-143. 
[44] Michael Polanyi, "Eine neue thermodynamische Folgerung aus der Quantenhypothese," Verhandlungen der Deutschen Physikalischen Gesellschaft 15 (1913) 156-161.

[45] Max Planck, Vorlesungen über Thermodynamik (Third edition. Lepzig: Veit, 1911).

[46] Max Planck, Vorlesungen über die Theorie der Wärmestrahlung (Second edition. Leipzig: Barth, 1913).

[47] Ulrich Hoyer, "Von Boltzmann zu Planck," Archive for History of Exact Sciences 23 (1980) 47-86.

[48] Olivier Darrigol, "Statistics and combinatorics in early quantum theory," Historical Studies in the Physical Science 19 (1988) 18-80.

[49] Max Planck, "Die gegenwärtige Bedeutung der Quantenhypothese für die kinetische Gastheorie," in David Hilbert, ed., Vorträge über die kinetische Theorie der Materie und der Elektrizität (Göttingen: Teubner, 1914), p. 14.

[50] Hugo M. Tetrode, "Die chemische Konstante der Gase und das elementare Wirkungsquantum," Annalen der Physik 38 (1912) 434-442.

[51] Otto Sackur, "Die Bedeutung des elementaren Wirkungsquantums für die Gastheorie und die Berechnung der chemischen Konstanten," Nernst Festschrift (Halle: Knapp, 1912), pp. $405-423$.

[52] Massimiliano Badino, "The odd couple: Boltzmann, Planck and the application of statistics to physics (1900-1913)," Annalen der Physik, 18 (2009) 81-101.

[53] Arnold Sommerfeld, "Das Plancksche Wirkungsquantum und seine allgemeine Bedeutung für die Molekularphysik," Physikalische Zeitschrift 12 (1911) 1057-1068.

[54] Niels Bohr, "Anwendung der Quantentheorie auf den Atombau. I. Die Grundpostulate der Quantentheorie," Zeitschrift für Physik 13 (1923) 117-165.

[55] Otto Sackur, "Die universelle Bedeutung des sogenannten elementaren Wirkungsquantums," Annalen der Physik 40 (1912) 67-86. 
[56] Arnold Sommerfeld, "Probleme der freien Weglänge," in David Hilbert, ed., Vorträge über die kinetische Theorie der Materie und der Elektrizität (Göttingen: Teubner, 1914), pp. 125-166.

[57] Otto Sackur, "Die 'Chemischen Konstante' der zwei- und dreiatomigen Gase," Annalen der Physik 40 (1912) 87-106.

[58] Otto Sackur, "Die Zustandgleichung der Gase und die Quantentheorie," Zeitschrift für Elektrochemie und angewandte Physikalische Chemie 20 (1914) 563-570.

[59] Heike Van Delft, Freezing Physics. Heike Kamerlingh Onnes and the quest for cold (Amsterdam: Koninklijke Nederlandse Akademie van Wetenschappen, 1912)

[60] Otto Stern, "Zur kinetischen Theorie des Dampfdrucks einatomiger fester Stoffe und über die Entropiekonstante einatomiger Gase," Physikalische Zeitschrift 14 (1913) 629632 .

[61] Otto Stern, "Zusammenfassender Bericht über die Molekulartheorie des Dampfdruckes fester Stoffe und ihre Bedeutung für die Berechnung chemischer Konstanten," Zeitschrift für Elektrochemie 25 (1919) 66-80.

[62] Enrico Fermi, "Sopra la teoria di Stern della costante assoluta dell'entropia di un gas perfetto monoatomico," Rendiconti della Accademia del Lincei 32 (1923) 395-398.

[63] Franco Rasetti, "La costante assoluta dell'entropia e le sue applicazioni," Il nuovo cimento 3 (1924) 67-86.

[64] Suman Seth, Crafting the Quantum. Arnold Sommerfeld and the Practice of Theory, 1890-1926 (Boston: MIT Press, 2010). 